\title{
Improving Cancer Therapy by Doxorubicin and Granulocyte Colony-Stimulating Factor: Insights from a Computerized Model of Human Granulopoiesis
}

\author{
V. Vainstein ${ }^{a, 1}$, Y. Ginosar ${ }^{a}$, M. Shoham ${ }^{b}$, A. Ianovski ${ }^{b}$ \\ A. Rabinovich ${ }^{b}$, Y. $\operatorname{Kogan}^{a}$, V. Selitser ${ }^{a}$, and Z. Agur ${ }^{a, b}$ \\ ${ }^{a}$ Institute for Medical Biomathematics (IMBM), POB 282 \\ Hate'ena 10, Bene Ataroth, 60991 Israel \\ ${ }^{b}$ Optimata Ltd, 7 Abba Hillel Silver St., Ramat-Gan, 52522 Israel
}

\begin{abstract}
Neutropenia is a significant dose-limiting toxicity of cancer chemotherapy, especially in dose-intensified regimens. It is widely treated by injections of Granulocyte ColonyStimulating Factor (G-CSF). However, optimal schedules of G-CSF administration are still not determined. In order to aid in identifying more efficacious and less neutropenic treatment protocols, we studied a detailed physiologically-based computer model of granulopoiesis, as affected by different treatment schedules of doxorubicin and/or Granulocyte ColonyStimulating Factor (G-CSF). We validated the model as evident from accurate prediction of clinical data on human granulopoiesis in healthy individuals and in doxorubicin-treated cancer patients, with or without G-CSF support. Based on our model, we suggest new G-CSF administration regimens. These regimens include reduced G-CSF doses, optimally timed post-chemotherapy. Application of these regimens can lead to minimization of G-CSF side effects, as well as more cost-effective and less myelotoxic protocols. Currently clinical trials are being designed in order to test these new treatment regimens.
\end{abstract}

Key words: mathematical modeling, granulopoiesis, chemotherapy, treatment optimization AMS subject classification: 92C50, 92C45, 35Q80

\footnotetext{
${ }^{1}$ Corresponding author. Email: vladimir@imbm.org
} 


\section{Introduction}

The concept of dose intensity in chemotherapy evolved during the last three decades from theory [9], [17] to proof of principle in clinical trials, where reduced interval between chemotherapy cycles resulted in improved survival in breast cancer and non-Hodgkin lymphoma patients [3], [4]. The rationale underlying dose intensification is based on dynamical considerations of tumor growth. First introduced by Goldie and Coldman in a simple logistic model of tumor cell proliferation, this concept is still valid in more complicated physiologically-based models of tumor growth [10].

The main dose-limiting toxicity resulting from chemotherapy intensification is neutropenia, which hinders the ability to administer the desired treatment intensity [14]. As a consequence, supporting growth factor therapies become a sine qua non condition of intensified protocols [3], [4]. One of the most widely used supporting agents is G-CSF (for review see Kuwabara et al. [12]) whose administration can be improved by predictive risk stratifying models [6]. However, the latter tools falls short of clarifying which schedule of G-CSF is optimal with respect to chemotherapeutic drugs. This question is evaluated in costly clinical trials by an inefficient trial-and-error method, which lead to controversial results [5], [11], [19], [21]. It should be noted that G-CSF can cause unpleasant side effects such as bone pain. In addition it is an expensive drug. Consequently, reducing G-CSF dosage has clinical and economic importance. Recently, we have put forward a new physiologically-based mathematical model of human granulopoiesis, which includes explicit description of the cellcycle transition phases [20]. The model, specifically designed to capture in detail multiple effects of G-CSF and chemotherapeutic drugs on human granulopoiesis, was retrospectively validated by a variety of published experimental data on human granulopoiesis [20].

The aim of the present work is to identify treatment strategies, which permit to optimize G-CSF administration schedule, while keeping myelotoxicity at a minimum and at the same time reducing G-CSF dose. To this end, we first studied the above granulopoiesis model in conjunction with a mathematical model for doxorubicin pharmacokinetics (PK) and pharmacodynamics (PD) and validated the comprehensive model by comparing its predictions to clinical results [2]. Subsequently, we compared the myelotoxicity predicted to be caused by different hypothetical schedules of G-CSF supporting treatment.

\section{Methods}

\subsection{Mathematical Model of Normal Granulopoiesis and G-CSF $\mathrm{PK} / \mathrm{PD}$}

The mathematical model of granulopoiesis [20] is formulated as a system of partial differential equations with delay describing the number of cells and the concentrations of G-CSF and GCSF receptors in the bone marrow and in the blood. The cell dynamics equations account for cell proliferation, including cell-cycle phase-transition, apoptosis and also transition between 
the different bone marrow compartments. The equations for G-CSF PK account for its production, its specific clearance by G-CSF receptor-bearing myeloid cells and its non-specific clearance. The equations for G-CSF PD describe its effects on bone marrow compartments of the myeloid line, including facilitation of proliferation, shortening of maturation and inhibition of apoptosis. A full description of derivation and validation of the mathematical model is published elsewhere [20].

The differential equations, which constitute the model, were transformed into difference equations and solved numerically. Simulation of the behavior of the whole system was performed using a computer program written in $\mathrm{C}++$ under Visual studio.NET environment.

\subsection{Mathematical model of doxorubicin pharmacokinetics (PK) and pharmacodynamics (PD)}

Doxorubicin is a widely used chemotherapeutic agent, whose mechanisms of action are believed to be DNA intercalation and topoisomerase II inhibition, resulting in inhibition of DNA replication [8]. Doxorubicin PK has linear dynamics, which can be fitted by a 3 compartments model [7]. Below we refer to these compartments as "drug compartments", to be distinguished from the cellular compartments of the bone marrow, which are described below and referred to as "compartments". Deep and shallow drug compartments represent tissues that exchange the drug with the blood. The effect of doxorubicin on granulopoiesis depends on its concentration in the bone marrow. We have modeled this process by drug compartments 4 and 5 (Figure 1), which represent free and bound drug, respectively. In this way we could achieve a prolonged effect of doxorubicin, long after it disappears from the blood, in accordance with experimental results [15].

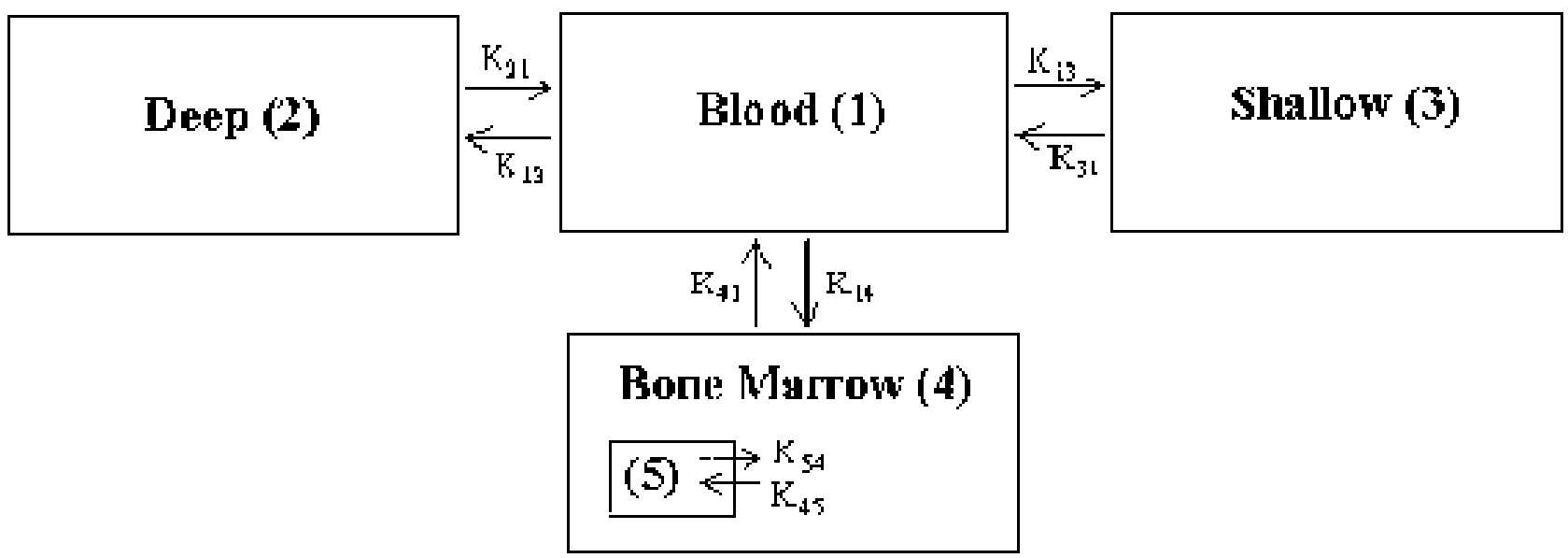

Figure 1: Schematic representation of doxorubicin pharmacokinetics (PK). Rectangles represent different drug compartments, while $k_{i j}$ stands for rate of transition from compartment $i$ to compartment $j$. 
The equations of doxorubicin PK/PD are presented in Appendix. The resultant model of doxorubicin $\mathrm{PK} / \mathrm{PD}$ successfully retrieved a variety of experimental data of different bolus and infusion schedules of doxorubicin administration [7] (data not shown).

\subsection{Parameter evaluation sequence}

Parameters of normal human granulopoiesis and G-CSF PK/PD, evaluated in Vainstein et al [20], were adopted in the simulations described in this work without any further modification. Doxorubicin PK/PD parameters were evaluated using an efficient search algorithm (eg., modified Powell's conjugate directions method) [1], [18]. Firstly, we evaluated doxorubicin PK parameters in compartments 1-3, based on experimental data [7]. Subsequently, we used the data from Bronchud et al [2] to adjust PK parameters in drug compartments 4 and 5, as well as PD parameters.

\subsection{Evaluation of neutropenia severity}

Two levels of neutropenia were calculated: 1) total neutropenia - under 40 percent of the baseline level; 2) grade 4 neutropenia - under 10 percent of the baseline level. These definitions apply to all the results below.

\section{Results}

\subsection{Evaluation of PD parameters of doxorubicin-induced myelo- toxicity}

In this section we retrieved parameters of doxorubicin myelotoxicity, and validated them on independent set of experimental data. Experimental data sets, used for these purposes, are described in Bronchud et al [2], where two groups of breast and ovarian cancer patients were treated with doxorubicin, with or without G-CSF (Table 1 protocols 1 and 2), and blood neutrophil counts were monitored for 43 days.

Doxorubicin PD parameters were retrieved based on neutrophil counts of patients treated by doxorubicin alone (Table 1 protocol 1). Comparison of the model output to experimental data is shown in Figure 2, left. The model is accurate in predicting neutrophil dynamics during recovery following doxorubicin administration. This is characterized by a low level of intrinsically produced G-CSF. Consequently, we are confident that the model extrapolations for low G-CSF dosage are valid.

The resulting parameters values were used without further modification to simulate neutropenia course in the group of patients treated by doxorubicin and G-CSF (Table 1 protocol 2 ), without any further parameter modification. As can be seen (Figure 2, right) the model can accurately predict neutrophil dynamics subjected to these two drugs. Additionally, our model predicts the nadir of bone-marrow proliferative cells between days 3 and 6 following doxorubicin administration, in accordance with in vivo experiments [4] (data not 

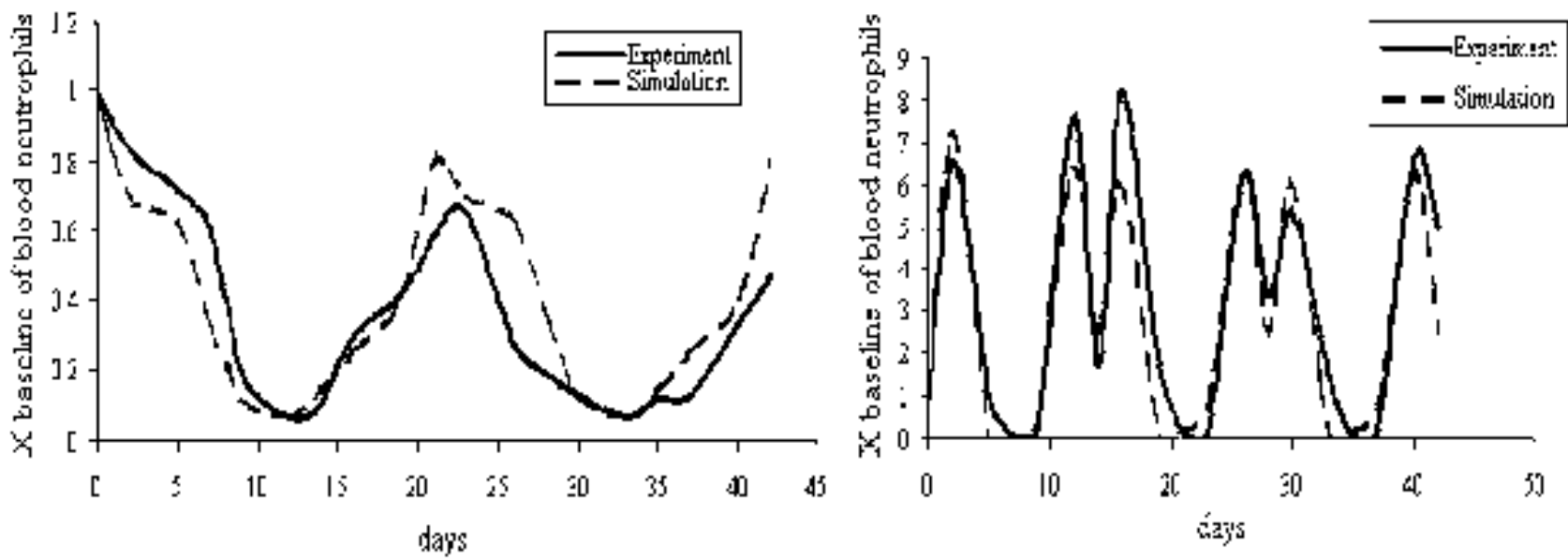

Figure 2: Validation of the model of doxorubicin-induced myelotoxicity. Simulation of blood neutrophil counts following doxorubicin administration was performed using the mathematical model of human granulopoiesis [20] in conjunction with doxorubicin PK/PD. The results were compared with clinical data from Bronchud et al [2]. (Left) Parameter evaluation: data of patients treated with doxorubicin alone were used for retrieval of the drug PD parameters. (Right) Model validation: blood neutrophil dynamics in patients treated with doxorubicin and G-CSF - the model predictions versus clinical data.

shown). These results validate the prediction accuracy of our models of granulopoiesis and doxorubicin-induced myelotoxicity.

\subsection{Optimization of combined G-CSF and doxorubicin therapy}

In this section we describe results of simulations of different hypothetical schedules of combined treatment of G-CSF and doxorubicin. Our aim is to identify regimen, which minimize both myelotoxicity and the dose of G-CSF, while maintaining the dose of doxorubicin.

\subsection{Varying onset of G-CSF support}

We have simulated several modifications to the original treatment protocol of Bronchud et al. [2] and evaluated their effect on granulopoiesis. The representative protocols and predicted duration of neutropenia are shown in Table 1.

Our model predicts higher myelotoxicity associated with an early onset of G-CSF treatment, i.e., on the day before doxorubicin (protocol 3 as compared to protocol 2 in Table 1). In contrast, a limited delay of G-CSF onset, following doxorubicin administration, shortens neutropenic duration (protocol 4 as compared to protocol 2 in Table 1). However, a delay of more than 4 days post-chemotherapy is inferior (protocol 5 as compared to protocol 4 in Table 1). 
Table 1. Protocols of combined dosorubicin and G-CSF therapy.

\begin{tabular}{|c|c|c|c|c|c|c|}
\hline \multirow[t]{2}{*}{ Protocol } & \multicolumn{2}{|c|}{ Doxorubicin } & \multicolumn{2}{|c|}{ G-CSF } & \multirow{2}{*}{$\begin{array}{c}\text { Total } \\
\text { neutropenia } \\
\text { duration }\end{array}$} & \multirow{2}{*}{$\begin{array}{c}\text { Grade } 4 \\
\text { neutropenia } \\
\text { duration }\end{array}$} \\
\hline & Days & istration & $\begin{array}{r}\text { Days } \\
\text { du }\end{array}$ & $\begin{array}{l}\text { cycle onset, dose and } \\
\text { ion of the cycles }\end{array}$ & & \\
\hline 1 & 0,21 & $75 \mathrm{mg} / \mathrm{m}^{2}$ & -- & -- & 24 & 7 \\
\hline 2 & $0,14,28$ & $75 \mathrm{mg} / \mathrm{m}^{2}$ & $1,15,29$ & $\begin{array}{l}700 \mu \mathrm{g} / \mathrm{d} \text { for } 7 \text { days } \\
350 \mu \mathrm{g} / \mathrm{d} \text { for } 4 \text { days }\end{array}$ & 17 & 10 \\
\hline 3 & $0,14,28$ & $75 \mathrm{mg} / \mathrm{m}^{2}$ & $-1,13,27$ & $\begin{array}{l}700 \mu \mathrm{g} / \mathrm{d} \text { for } 7 \text { days } \\
350 \mu \mathrm{g} / \mathrm{d} \text { for } 4 \text { days }\end{array}$ & 22 & 15.5 \\
\hline 4 & $0,14,28$ & $75 \mathrm{mg} / \mathrm{m}^{2}$ & $4,18,32$ & $\begin{array}{l}700 \mu \mathrm{g} / \mathrm{d} \text { for } 4 \text { days } \\
350 \mu \mathrm{g} / \mathrm{d} \text { for } 4 \text { day s }\end{array}$ & 6.5 & 0 \\
\hline 5 & $0,14,28$ & $75 \mathrm{mg} / \mathrm{m}^{2}$ & $5,19,33$ & $\begin{array}{l}700 \mu \mathrm{g} / \mathrm{d} \text { for } 3 \text { days } \\
350 \mu \mathrm{g} / \mathrm{d} \text { for } 4\end{array}$ & 10 & 0 \\
\hline 6 & $0,14,28$ & $75 \mathrm{mg} / \mathrm{m}^{2}$ & $1,15,29$ & $100 \mu \mathrm{g} / \mathrm{d}$ for 11 days & 16 & 5.5 \\
\hline 7 & $0,14,28$ & $75 \mathrm{mg} / \mathrm{m}^{2}$ & $4,18,32$ & $100 \mu \mathrm{g} / \mathrm{d}$ for 8 days & 7 & 0 \\
\hline
\end{tabular}

The protocols and their simulation results are displayed, using granulopoiesis model [20] as well as doxorubicin and G-CSF PK/PD (Appendix). Protocols 1 and 2 are described in Bronchud et al [2], while all other protocols are hypothetical. Doxorubicin was administered as intravenous bolus injection. We assumed 1.7 squared meters as an average body surface area for calculation of the total doxorubicin dose. G-CSF was administered as a continuous intravenous infusion. Day 0 was the day of first administration of doxorubicin. For calculation of neutropenia duration, simulations were run up to day $50 . \mathrm{mg} / \mathrm{m} 2$ indicates milligrams per meter squared of patient's body surface; $\mu \mathrm{g} / \mathrm{d}$, micrograms per day. 


\subsection{Minimizing G-CSF Dosage}

We have checked whether G-CSF daily dosage can be reduced in protocol 2 (original intensified protocol of Bronchud et al. [2]; Table 1) and protocol 4 (delayed onset of G-CSF treatment; Table 1). Simulation results of two representative protocols are shown in Table 1. Based on our simulations, we predict that the total dose of administered G-CSF can be reduced more than 5 times without increasing the severity of neutropenia (protocols 6 and 7, as compared to protocols 2 and 4, respectively, Table 1). Further reduction of G-CSF dosage led to inferior results.

Table 2. Dosorubicin PK parameter values.

\begin{tabular}{|cc|}
\hline Parameter & ${\text { Value }\left(\text { hour }^{-1}\right)}^{-1}$ \\
\hline $\mathrm{k}_{12}$ & 14.768 \\
\hline $\mathrm{k}_{21}$ & 0.096 \\
\hline $\mathrm{k}_{13}$ & 5.035 \\
\hline $\mathrm{k}_{31}$ & 1.704 \\
\hline $\mathrm{k}_{14}$ & 0.1 \\
\hline $\mathrm{k}_{41}$ & 8.215 \\
\hline $\mathrm{k}_{45}$ & 0.6 \\
\hline $\mathrm{k}_{54}$ & 0.025 \\
\hline $\mathrm{k}_{\mathrm{e}}$ & 6.129 \\
\hline
\end{tabular}

Doxorubicin PK parameters evaluated from human experimental data [7], as described in "Methods". Meanings of the parameters appear in Figure 1.

\section{Discussion}

Recently, superiority of dose intensified chemotherapy protocols, with granulopoietic supporting factors (eg, G-CSF) over standard regimens, has been demonstrated in large randomized prospective clinical trials in different types of cancer. Nevertheless, the optimal GCSF administration protocol, with respect to mortality, morbidity and cost-effectiveness are 
still to be determined. The number of possible protocols of administration of 3-4 chemotherapeutic drugs and one supportive agent is huge, and it is impossible to evaluate in clinical trials all the different possibilities. Consequently, "rules of thumb" or more sophisticated methods of determination of the most promising protocols should be applied. "Rules of thumb" appear to fail in such a question as whether or not and by how long to delay the initiation of G-CSF support post-chemotherapy, leading to controversial results [5], [11], [19], [21]. In this work we applied a physiologically-based mathematical model of human granulopoiesis [20] in conjunction with doxurubicin PK/PD model in order to evaluate improved protocols of chemotherapeutic and myelo-supportive treatment. Concerning the timing of G-CSF support, our simulations show that the optimal delay is 4 days after doxorubicin administration. Both shorter and longer delays appear to be inferior, while initiation of GCSF treament before chemotherapy is even more toxic. We believe that the reason for these results is an inter-play between two driving forces. From one side, G-CSF drives the mitotic cells into proliferation [16], where they are more susceptible to doxorubicin [15], justifying delay of G-CSF administration. From the other side, such a delay can compromise efficiency of G-CSF-induced stimulation of myeloid cells leading to delay in neutrophil recovery. Obviously, the choice of the optimal timing cannot be made by simple intuition, while application of a mathematical model gives biologically reasonable and experimentally verifiable results.

With respect to G-CSF dosage, our analysis suggests that when doxorubicin monotherapy is considered, G-CSF dose can be reduced by a factor of 6 , without aggravating neutropenia, thus enabling to propose more cost-effective administration schedules for this expensive drug, and hoping to minimize its significant side effects. We conclude that a physiologically-based mathematical modeling to be a promising strategy for rationalizing the selection of few treatment schedules between the large number of potential ones. The theoretically selected schedules are to be validated in a small number of focused clinical trials.

\section{Appendix: Mathematical model of doxorubicin PK and PD}

The PK of doxorubicin described in Figure 1 is represented by a set of the following ordinary differential equations:

$$
\begin{gathered}
\frac{d A_{1}}{d t}=-\left(k_{e}+k_{12}+k_{13}+k_{14}\right) A 1+k_{21} A_{2}+k_{31} A_{3}+k_{41} A_{4} \\
\frac{d A_{2}}{d t}=k_{12} A_{1}-k_{21} A_{2} \\
\frac{d A_{3}}{d t}=k_{13} A_{1}-k_{31} A_{3} \\
\frac{d A_{4}}{d t}=k_{14} A_{1}-\left(k_{41}+k_{45}\right) A_{4}+k_{54} A_{5}
\end{gathered}
$$




$$
\frac{d A_{5}}{d t}=k_{45} A_{4}-k_{54} A_{5},
$$

where $A_{i},(i=1,2, \ldots, 5)$ is the amount of doxorubicin in drug compartment $i, k_{i j}$ is a transition constant from drug compartment $i$ to drug compartment $j$. Here $\gamma$ represents the fraction of free drug in the blood, which estimated value is $0.85, k_{e}$ is elimination rate. Parameters were evaluated as described in Methods. The resultant values are shown in Table 2.

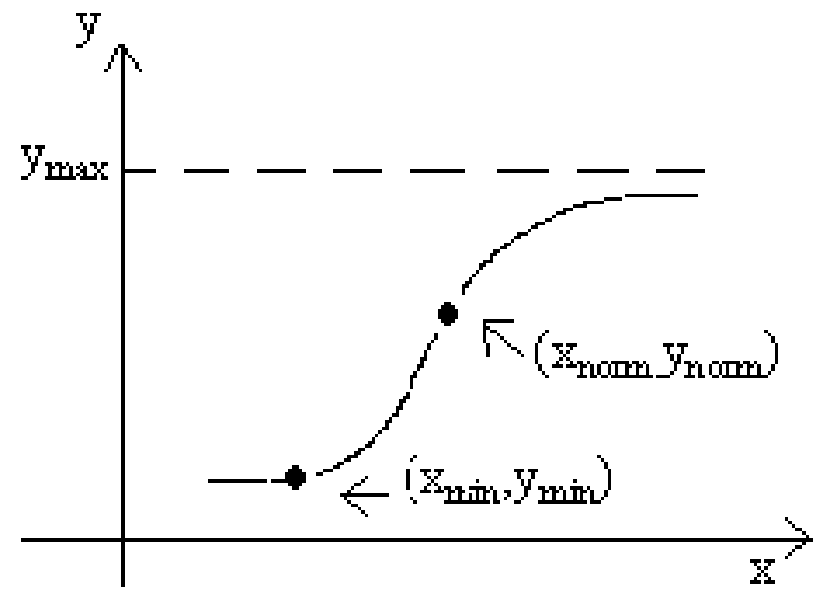

Figure 3: PD of doxorubicin-induced neutropenia. General form of a sigmoid function modeling doxorubicin effect on cell population: here $y(x)$ represents killing rate of a certain type of cells (e.g., myelocytes in S-phase) for some drug concentration $x(t)$ in drug compartment 5 (see Figure 1 ); $x_{\min }$ and $y_{\min }$ were set zero values.

The PD of doxorubicin was modeled as killing rates of bone-marrow cells dependent on the bone marrow drug concentration through the sigmoid function of drug concentration in drug compartment 5 as shown in Figure 3. This sigmoid function is implemented piecewise by two hyperbolae of the form $y=A+B /(x+C)$. Here $y(x)$ represents for some drug concentration $\mathrm{x}$. We imposed first-order continuity in the point of ligature and zero value for $x=0$. These function's coefficients are determined by 3 values - asymptotic maximal value and coordinates of the ligature point (see Figure 3). We have introduced PD effects into each of the mitotic compartments of bone marrow, such that the three parameters of the sigmoid function could be different in each compartment. A different effect was applied to resting and cycling cells, based on Kwok and Twentyman [13]. An additional effect, inversely related to age, was introduced in the post-mitotic compartment.

Acknowledgements. The authors wish to thank Chai Foundation for financial support. 


\section{References}

[1] R.P. Brent. Algorithms for minimization without derivatives. Englewood Cliffs, Prentice-Hall, NJ, 1973.

[2] M.H. Bronchud, A. Howell, D. Crowther, P. Hopwood, L. Souza, T.M. Dexter. The use of granulocyte colony-stimulating factor to increase the intensity of treatment with doxorubicin in patients with advanced breast and ovarian cancer. Br. J. Cancer, 60 (1989), 121-125.

[3] M.L. Citron, D.A. Berry, C. Cirrincione, C. Hudis, E.P. Winer, W.J. Gradishar, N.E. Davidson, S. Martino, R. Livingston, J.N. Ingle, E.A. Perez, J. Carpenter, D. Hurd, J.F. Holland, B.L. Smith, C.I. Sartor, E.H. Leung, J. Abrams, R.L. Schilsky, H.B. Muss, L. Norton. Randomized trial of dose-dense versus conventionally scheduled and sequential versus concurrent combination chemotherapy as postoperative adjuvant treatment of node-positive primary breast cancer: first report of Inter-group Trial C9741/Cancer and Leukemia Group B Trial 9741. J. Clin. Oncol., 21 (2003), 1431-1439.

[4] B. Coiffier. Increasing chemotherapy intensity in aggressive lymphomas: a renewal? J. Clin. Oncol, 21 (2003), 2457-2459.

[5] J. Crawford, H. Kreisman, H. Garewal, S.E. Jones, D. Shoemaker, M.R. Pupa, S. Armstrong, D. Tomita, G. Dziem. Impact of Filgrastim schedule variation on hematopoietic recovery post-chemotherapy. Ann. Oncol., 8 (1997), 1117-1124.

[6] C.T. Dang, M.N. Fornier, C.A. Hudis. Risk models for neutropenia in patients with breast cancer. Oncology (Huntingt), 17 (2003), 14-20.

[7] S. Eksborg, H.S. Strandler, F. Edsmyr, I. Naslund, P. Tahvanainen. Pharmacokinetic study of i.v. infusions of adriamycin. Eur. J. Clin. Oncol., 28 (1985), 205-212.

[8] J.G. Hardman, L.E. Limbird. The pharmacological basis of therapeutics. McGraw-Hill, 2001.

[9] W.M. Hryniuk. Average relative dose intensity and the impact on design of clinical trials. Semin. Oncol., 14 (1987), 65-74.

[10] Y. Kogan, B. Ribba, K. Marron, N. Dahan, V. Vainstein, Z. Agur. Residual nonHodgkin's lymphoma disease: towards a computationally supported treatment improvement. Math. Model. Nat. Phenom., 2 (2007), No. 3, in press.

[11] G. Koumakis, M. Vassilomanolakis, V. Barbounis, E. Hatzichristou, S. Demiri, G. Plataniotis, F. Pamouktsoglou, A.P. Efremidis. Optimal timing (preemptive versus supportive) of granulo-cyte colony-stimulating factor administration following high-dose cyclophosphamide. Oncology, 56 (1999), 28-35. 
[12] T. Kuwabara, S. Kobayashim, Y. Sugiyama. Pharmacokinetics and pharmacodynamics of a recombinant human Granulocyte Colony-Stimulating Factor. Drug Met. Rev., 28 (1996), 625-658.

[13] T.T. Kwok, P.R. Twentyman. The relationship between tumour geometry and the response of tumour cells to cytotoxic drugs - an in vitro study using EMT6 multicellular spheroids. Int. J. Cancer, 35 (1985), 675-682.

[14] R.C. Leonard, D. Miles, R. Thomas, F. Nussey. UK Breast Cancer Neutropenia Audit Group. Impact of neutropenia on delivering planned adjuvant chemotherapy: UK audit of primary breast cancer patients. Br. J. Cancer, 89 (2003), 2062-2068.

[15] H.-P. Lohrmann, W. Schreml. Cytotoxic drugs and the granulopoietic system. Recent Results in Cancer Research, 8 (1982), 11-222.

[16] B.T. Lord, M.H. Bronchud, S. Owens, J. Chang, A. Howell, L. Souza, T.M. Dexter. The kinetics of human granulopoiesis following treatment with granulocyte colony-stimulating factor in vivo. Proc. Natl. Acad. Sci. USA, 88 (1989), 9499-9503.

[17] L. Norton, R. Simon. The Norton-Simon hypothesis revisited. Cancer Treat. Res., 70 (1986), 163-169.

[18] W.H. Press, B.P. Flaunery, S.A. Teukolsky, W.T. Vetterling. Numerical recipes in C: The art of scientific computing. Cambridge University Press, Cambridge, 1993.

[19] H. Soda, M. Oka, M. Fukuda, A. Kinoshita, A. Sakamoto, J. Araki, S. Fujino, N. Itoh, K. Watanabe, T. Kanda, M. Nakano, K. Hara. Optimal schedule for administering granulocyte colony-stimulating factor in chemotherapy-induced neutropenia in non-small-cell lung cancer. Cancer Chemother. Pharmacol., 38 (1996), 9-12.

[20] V. Vainstein, Y. Ginosar, M. Shoham, D.O. Ranmar, A. Ianovski, Z. Agur. The complex effect of granulocyte colony-stimulating factor on human granulopoiesis analyzed by a new physiologically-based mathematical model. J. Theor. Biol., 234 (2005), 311-27.

[21] M.A. Weiser, S. O'Brien, D.A. Thomas, S.A. Pierce, T.P. Lam, H.M. Kantarjian. Comparison of two different schedules of granulocyte-colony-stimulating factor during treatment for acute lymphocytic leukemia with a hyper-CVAD (cyclophosphamide, doxorubicin, vincristine, and dexamethasone) regimen. Cancer, 94 (2002), 285-291. 